\title{
On time boundary of functional metal properties maintainance under extreme conditions
}

\author{
A.Ya. Uchaev, N.I. Selchenkova ${ }^{a}$, E.V. Kosheleva, and L.V. Zhabyka \\ Russian Federal Nuclear Center - VNIIEF, Nizhni Novgorod Region, Sarov 607188, Russia
}

\begin{abstract}
There are established the boundaries of conserving functional properties of metals at dynamic failure under extreme conditions (longevity range $\mathrm{t} \sim 10^{-6} \div 10^{-10} \mathrm{~s}$ ). The methods of non-linear physics made it possible to determine universal attributes of non-equilibrium systems evolution conditioned by collective effects and phenomena of self-organization in the appearing dissipative structures. In the paper there is presented a universal failure surface that allows simulation of metals behavior under laboratory conditions and forecast of time boundary of functional properties conservation.
\end{abstract}

\section{Introduction}

Today quite acute is the knowledge of material behavior under extreme conditions, in highly non-equilibrium states when the value of energy variation from the equilibrium state is compatible to the energy of phase transition, for example. As a rule, relaxation of highly non-equilibrium states is accompanied by dynamic destructive processes [1-4].

At small degrees of deviation from equilibrium the relaxation processes are determined by kinetics mechanisms that are described by the classic kinetics instrument.

For example, for gases when chaotization occurs, one can go over to a single-part distribution function. In the failure physics in a quasi-static longevity range the time interval up to failure is determined by the time of critical fluctuation formation, for example, energy leading to destruction of a part of atomic bondings. Above-mentioned processes are ergodic [2].

At higher degrees of deviation from the equilibrium state relaxation is characterized not by the processes typical for initial (quasi-stationary) relaxation processes but by the newly occurred relaxation processes having hierarchic scale-time subordination.

The phenomenon of dynamic failure refers just to this type of relaxation processes [2].

From the point of view of failure process forecast and time boundary determination for functional properties maintainance not only the final phase is of significance but the preceding history of the process [1-4] as well. Thus, acute is the investigation of the entire sequence of events preceding the threshold state as well as determination of time boundary of functional properties maintainance for a set of metals under extreme conditions. This is just the goal of the current paper.

\section{Results and discussion}

As a result of investigations performed it was demonstrated that the resistance of the body against the external effect is offered by dissipative structure, for example,

a e-mail: n-selchenkova@expd.vniief.ru cascade of failure centers. The evolution of micro- and meso-scopic defects in the phenomenon of dynamic failure is critical in the behavior of solid bodies under the effect of high-power pulses of radiation within the $t \sim 10^{-6} \div 10^{-11}$ longevity range (rate of temperature variation $\mathrm{d} T / \mathrm{d} t \sim$ $10^{6} \div 10^{12} \mathrm{~K} / \mathrm{s}$, range of initial temperatures $T_{0} \sim 4 K \div 0.8 T_{\mathrm{m}}$, where $T_{\mathrm{m}}$ - melting temperature) [2-4].

Earlier there was obtained spectral distribution of failure centers over sizes, formed in the course of dynamic failure process that takes the form:

$$
N_{i}\left(D_{i}\right) \sim D_{i}^{-\alpha}
$$

where $N_{i}$ - number of failure centers of size $D_{i}$ and $\alpha$ - fractal dimension [1-4].

Failure centers cascade is a self-similar cluster, i.e.

$$
D_{i}=q D_{i-1}=q^{i} L
$$

$L$ - system size.

In non-linear dissipative media - the metals in the course of dynamic failure being such media - there takes place the decrease of the number of degrees of freedom. It means that in the system there occurs self-organization of structural elements characterized by large-scale correlations. The order parameter characterizes transition from non-correlated state of substance to the correlated one. Density of failure centers $f(t)$ depending on time is the order parameter at the failure threshold when the continuity of the failed body varies. Essential increase of the density of failure centers $f(t)$ takes place at the final stage of the failure process at times $t \sim 0.9 \cdot t_{r}$, where $t_{r}$ - failure duration [4].

The rate of accumulating failure for processes with different pressure amplitude $P_{i}$ will look like: $d w / d t=$ $P^{\gamma} F\left(w, P_{1}\right) / P_{1}^{\gamma}=P^{\gamma} F\left(w, P_{2}\right) / P_{2}^{\gamma}=\ldots$, i.e. we have $d w / d t=$ const; $F\left(w, P_{i}\right)$, function depending on the failure rate $w$ and pressure $P_{i}$ [4].

According to definition, the failure rate is $w=V_{r} / V_{0}$, $V_{0}$ - initial body volume, $V_{r}$ - body failed volume, $V_{r}=$ $\sum N_{i} V_{i}, V_{i}$ - the volume of a pore with a size $D_{i}$ or $V_{r}=\sum N_{i} D_{i}^{3}$, as far as $N_{i} \sim D_{i}^{-\alpha}, V_{r}=\sum D_{i}^{3-\alpha}$. The rate of accumulating failure is expressed by:

$$
d w / d t=\sum d D(t)_{i}^{3-\alpha} / d t
$$

This is an Open Access article distributed under the terms of the Creative Commons Attribution License 2.0, which permits unrestricted use, distribution, and reproduction in any medium, provided the original work is properly cited. 

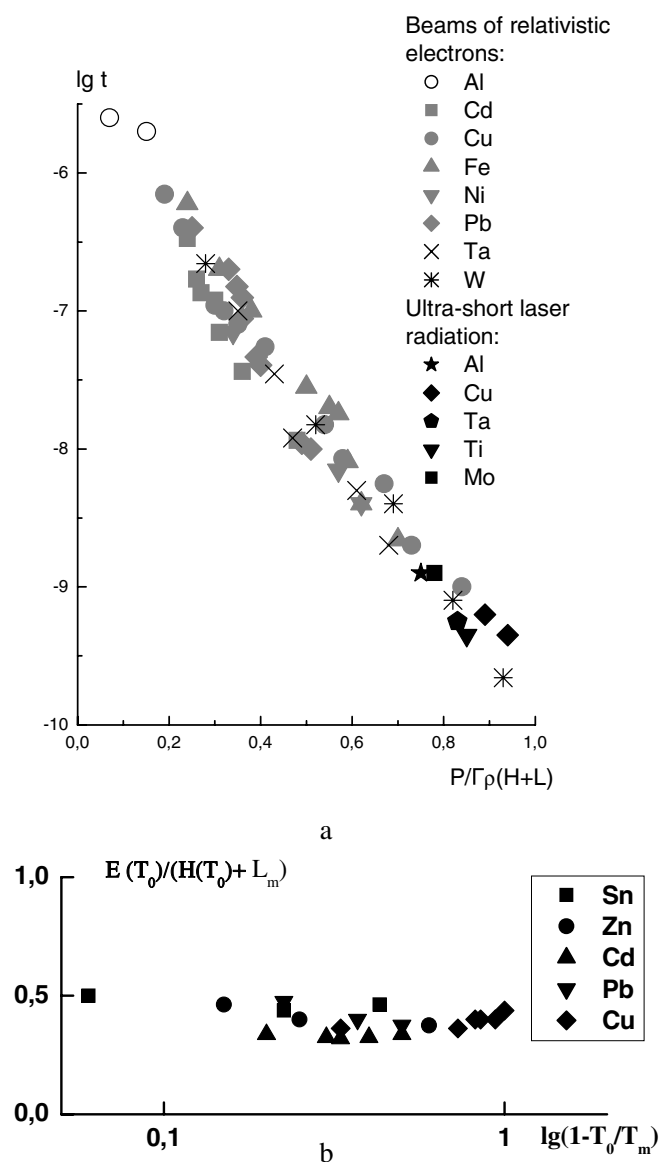

Fig. 1. Dependences of dynamic failure process: a - temporary; $\mathrm{b}$ - temperature.

To describe basic types of critical behavior one can use simple models demonstrating the principal ways of transition to self-organization $[1,2]$. From the property of selfsimilarity of the process of dynamic failure there follows the relation between the amplitude of critical pressure $\mathrm{P}$ and material life that is defined as

$$
P(t)^{\gamma} t=\text { const, }
$$

where $\gamma \approx 3.8[1,2]$. This is just the condition that defines the possibility of simulating the process of dynamic failure under laboratory conditions at scaling the duration of the real process [2].

Basing on the found temperature-scale regularities (special for each metal) there were obtained by conditionally calculation-theoretical method the following data on the boundary of failure within the $t \sim 10^{-6} \div 10^{-10} \mathrm{~s}$ longevity range and within the $T_{0} \sim 4 \mathrm{~K} \div 0.8 T_{m}$ range of initial temperature: time dependence (Fig. 1, a) and temperature dependence (Fig. 1, b).

Figure 1, a gives the time dependence of the process of dynamic failure for a set of metals under the effect of relativistic electron beams (REB) and short pulses of laser radiation in $\mathrm{t}$ coordinates, dynamic invariant $I=P_{c r}(t) / \Gamma \rho\left(H+L_{m}\right)\left(P_{c r}-\right.$ critical pressure leading to failure, $\Gamma$ - Gruneisen parameter, $\rho$ - material density, $H$ - enthalpy and $L_{m}$ - heat of fusion). Let us mention that critical density of absorbed energy leading to failure of, for example, $\mathrm{Ti}, \mathrm{Al}, \mathrm{Ta}, \mathrm{Cu}$ metals under the effect of short laser radiation pulses and REB coincides (Fig. 1, a).
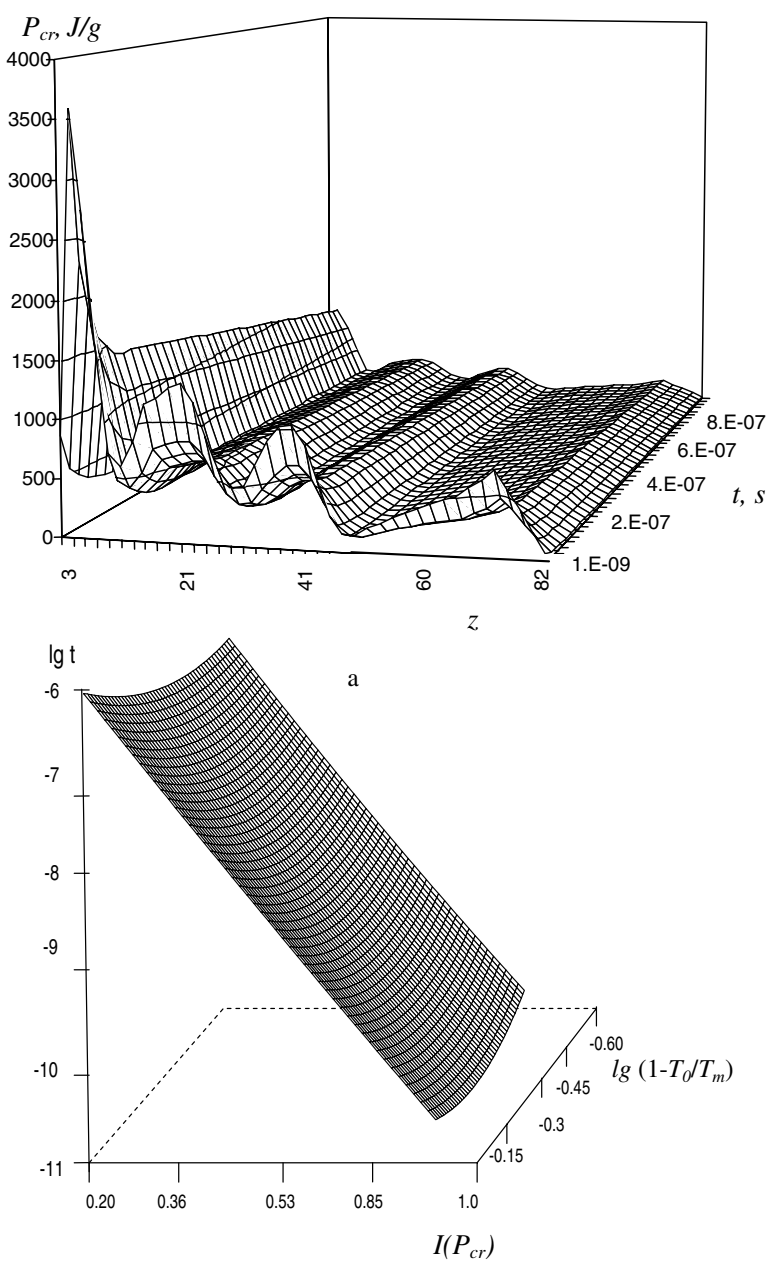

Fig. 2. Failure surfaces: a - in $t, P_{c r}, z$ coordinates; $\mathrm{b}-$ in $t, I\left(P_{c r}\right)$, $\lg \left(1-T_{0} / T_{m}\right)$ coordinates.

With the use of dynamic invariants and conclusions of the given work there is constructed the failure surface (Fig. 2, a) in the coordinates of longevity t, critical pressure $P_{c r}$, atomic number of the element in the periodic table $\mathrm{z}$, that describes the boundary above which there is the failure region and which demonstrates periodic behavior of critical pressure $P_{c r}$ depending on the periodic number of element $\mathrm{z}$. Any point of the surface $R(t, P, z)$, where $t \sim 10^{-6} \div 10^{-10} \mathrm{~s}$ defines for any metal with different $\mathrm{z}$ the boundary above which there is the region of failure and below which - region of resistance. Figure 2 , b presents the failure surface in universal coordinates $t, I\left(P_{c r}\right), \lg (1-$ $\left.T_{0} / T_{m}\right)$ that is single for all metals which quantitatively makes it possible to simulate adequately the behavior of metals in the extreme state at laboratory conditions as well as to forecast the behavior of non-investigated metals under extreme conditions.

\section{Conclusions}

The single mechanism of the process of metals dynamic failure - loss of system (sample) continuity due to clusterization of the cascade of failure centers, the same order parameter and similar spatial dimension where the process takes place - proves the possibility of forecasting metals behavior under extreme conditions. The above-said 
defines scale-invariant properties of dissipative structures behavior and conditions universal behavior of metals under the phenomenon of dynamic failure at different time intervals with different amplitude-time characteristics of external effect. The universal phase surface of metals failure makes it possible to adequately simulate the behavior of substance under extreme conditions through studying the behavior of samples in laboratory conditions and forecast the time boundary of metals functional properties conservation under extreme conditions. As a result of undertaken researches it is shown that physical nature of metals life under extreme conditions is defined by the time of critical concentration formation of failure center cascades that constitute percolation cluster.

\section{References}

1. R. I. Il'kaev, A. Ya. Uchaev, S. A. Novikov, N.I. Zavada, L. A. Platonova, N. I. Selchenkova, DAN 384, 3 (2002)

2. V.T. Punin, A. Ya. Uchaev, S. A. Novikov, E.V. Kosheleva, L. A. Platonova, N. I. Selchenkova, N.A. Yukina, DAN 393, 3 (2003)

3. E. K. Bonyushkin, N. I. Zavada, S. A. Novikov, A. Ya. Uchaev, Kinetics of dynamic metals failure under the mode of pulse volume heat-up (Sarov, RFNC-VNIIEF Press, 1998)

4. R.I. Il'kaev, V.T. Punin, A. Ya. Uchaev, E.V. Kosheleva, L. A. Platonova, N. I. Selchenkova, A.S. Konkin, Nuclear Physics and engineering 1, 2 (2010) 